\title{
Lead Placement Frank
}

National Cancer Institute

\section{Source}

National Cancer Institute. Lead Placement Frank. NCI Thesaurus. Code C71118.

An electrocardiogram (ECG) lead placement for determining 3 orthog onal components $X$ (right to left direction), $Y$ (foot to head direction) and Z (back to front direction) of the heart. For this method a minimum of 4 electrodes are needed that represent the right arm, left arm, left leg and back. However, ususally 7 electrodes are used to avoid dependence on the dipole location and facilitate interpretation. 